\title{
APRENDIZAGEM OBSERVACIONAL, FORMAÇÃO E EXPANSÃO DE CLASSES DE ESTÍMULOS EQUIVALENTES
}

\author{
STIMULUS EQUIVALENCE CLASS FORMATION AND EXPANSION VIA \\ OBSERVATIONAL LEARNING
}

\author{
Celso Goyos, Adriana Aparecida Tambasco Piccolo, Gisele Porto \\ \& TALES C. LaZARIN \\ UNIVERSIDADE FEDERAL DE SÃO CARLOS, BRASIL.
}

\begin{abstract}
RESUMO
Este estudo teve como objetivo investigar se um procedimento de ensino por modelação pode produzir discriminações condicionais, formar classes de estímulos equivalentes, e expansão de classes, em seis estudantes universitários. Foram ensinadas discriminaçōes condicionais entre cinco conjuntos de estímulos, através de tarefas de escolha de acordo com o modelo. Foram usados como estímulos desenhos de objetos comuns produzidos em linhas pretas sobre fundo branco. As relaçôes BA foram ensinadas da maneira tipicamente descrita pela literatura, sem modelo, seguidas de ensino das relações CA, com modelo. No ensino das relações BA, os participantes escolhiam os estímulos de comparação e recebiam conseqüências diferenciais para escolhas corretas e incorretas. No ensino de CA, assim como no de DA e EA, os participantes foram instruídos a observar um demonstrador que selecionava os estímulos de comparação e recebia consequiências diferenciais por suas escolhas. Após o ensino das relaçôes BA CA, foram introduzidos os testes para verificar a emergência de simetria, transitividade e equivalência. Todos os participantes mostraram formação de classes equivalentes e cinco deles expansão de classes para os estímulos D e E. Discute-se se os resultados produzidos foram função exclusiva do ensino por modelação, ou uma combinação desses com efeitos seqüenciais do ensino individual e direto da primeira relação condicional.
\end{abstract}

Palavras-chave: ensino por modelação, equivalência de estímulos, discriminações condicionais, escolha de acordo com o modelo, aprendizagem observacional

\begin{abstract}
This study investigated conditional discrimination acquisition, stimulus class formation and stimulus class expansion through a modeling procedure. Six college students were taught conditional discriminations with five stimulus sets. Stimuli were black line drawings of common objects against white backgrounds. BA relations were taught individually, in a manner similar to that typically described by the literature, without modeling, followed by the teaching of CA relations, taught through a modeling procedure. During the teaching of BA relations, correct and incorrect comparison selections were followed by differential reinforcement. During CA, subsequent DA relations and EA relations training, subjects were instructed to observe a model that actually made the selections and received differential reinforcement for that. After the teaching of BA and CA relations, symmetry, transitivity and equivalence tests were introduced, followed by DA and EA teaching and tests for equivalence class expansion. All participants showed equivalence class formation and all but one participant showed expanded classes to include stimulus sets $\mathrm{D}$ and $\mathrm{E}$ into the previously formed class. Whether the observed results were an exclusive function of modeling or a combination of modeling and sequential effects of the directing teaching of BA requires further investigation.
\end{abstract}

Key words: modeling, stimulus equivalence, conditional discriminations, observational learning

Grande parte da aprendizagem da criança e do adulto ocorre através da observação de um modelo. A aprendizagem por observação, ou aprendizagem observacional é a habilidade de adquirir novas respostas como resultado da observação do comportamento de um modelo,

\footnotetext{
O trabalho é parte da tese de doutorado e de iniciação científica, respectivamente, da segunda autora, e do terceiro e quarto autores. Agradecemos o apoio da FAPESP através dos processos 00/09627-6; 00/07752-8; 00/09502; 00/09501-2, e ao CNPq pela bolsa produtividade ao primeiro autor.

Correspondência para Prof. Dr. Celso Goyos e-mail: celsogoyos@hotmail.com.
} 
e constitui-se em uma estratégia importante para promover ensino e aprendizagem eficaz e econômica em sala de aula (Browder, Schoen, \& Lentz, 1986). A aprendizagem observacional pode também ser definida como o resultado do ensino por modelação. É de senso comum dizer que o comportamento de uma criança em sala de aula pode ocorrer sob o controle do comportamento de outras crianças, assim como acontece sob o controle do comportamento do professor e do ambiente físico. É comum ouvir que o comportamento inadequado de um aluno foi desencadeado pelo comportamento inadequado de outras crianças. Da mesma forma, é comum ouvir relatos sobre o comportamento de uma criança que é reforçado pela professora e também leva os comportamentos de outras crianças a ficar sob o controle das mesmas condições. A aprendizagem por observação, ou aprendizagem observacional torna-se uma estratégia rápida de integrar o indivíduo em um ambiente novo.

A aprendizagem por observação tem sido particularmente útil na construção de repertórios comportamentais mais sofisticados em indivíduos com deficiência mental, principalmente no ensino do comportamento verbal (Lovaas, Beberich, Perloff, \& Schaeffer, 1966). Como condição de ensino para esses indivíduos, a estratégia de modelação pode ser essencial, uma vez que, com repertório comportamental limitado o indivíduo enfrenta inúmeras demandas de aprendizagem de comportamentos inéditos para ser funcional em um ambiente complexo de integração. No entanto, o indivíduo com deficiência mental parece não aprender tão rapidamente quando o ensino é realizado por modelação (Baer \& Sherman, 1964; MacDonald, Dixon, \& LeBlanc, 1986; Striefel \& Wetherby, 1973).
A definição de aprendizagem observacional, a partir de Whitehurst (1978, apud Ruggles \& LeBlanc, 1982) expandiu a noção de que comportamento do observador devesse ser topograficamente semelhante ou idêntico ao do demonstrador, para incluir também os casos em que o comportamento do demonstrador e do observador são controlados pelos mesmos estímulos discriminativos, ou estímulos contextuais, ou ainda pelos resultados daqueles comportamentos que são parte de uma mesma classe funcional (Ruggles \& LeBlanc, 1982).

MacDonald et al. (1986) expandiram ainda mais esta definição, englobando os estímulos que fazem parte de uma mesma classe de equivalência. Esta expansão tornou a definição de aprendizagem observacional mais interessante à medida que permite vislumbrar uma abrangência comportamental muito maior por meio do ensino por modelação. No entanto, ela traz um problema adicional para a área, pois em equivalência de estímulos, o indivíduo aprende relações simbólicas entre conjuntos de eventos, ou seja, exige-se do indivíduo que seja capaz de abstrair as relaçôes corretas entre estímulos (Wright, Cook, Rivera, Sands, \& Delius, 1988). Sidman e Tailby (1982) identificaram as propriedades relevantes para determinar quando estímulos se tornam membros equivalentes de uma mesma classe. O paradigma de equivalência de estímulos, desde então, foi objeto de vários refinamentos teóricos e experimentais (Sidman, 1990; 1994; 2001), e tem sido instrumental para o ensino de uma série de comportamentos simbólicos relativamente sofisticados para indivíduos com deficiência mental (Sidman, 1971; Rossit, Goyos, Araújo, \& Nascimento, 2001; Silva, Ferreira \& Goyos, 2001) e com dificuldades de aprendizagem (de 
Rose, Souza, \& Hanna, 1996; de Rose, Souza, Rossito, \& de Rose, 1989).

$\mathrm{O}$ paradigma de equivalência de estímulos, por ser muito bem refinado experimentalmente (Green \& Saunders, 1998; Sidman, 1994; 2001), e tratar de comportamentos altamente complexos, oferece um método para a investigação sistemática do contraste entre ensino individual e ensino por modelação (MacDonald et al., 1986). Além disso, por ter enorme potencial de aplicabilidade prática no ensino (Stromer, Mackay, \& Stoddard, 1992), e por muitas vezes os ambientes escolares não oferecerem condições de ensino individualizado, é importante que se considerem estratégias práticas eficazes para o ensino em pequenos grupos (MacDonald et al.).

Pelas razões acima mencionadas, é importante que se compreenda melhor como indivíduos com desenvolvimento típico e com deficiência mental podem se beneficiar do ensino de discriminações condicionais por modelação.

MacDonald et al. (1986) investigaram a aprendizagem observacional de tarefas de escolha de acordo com o modelo com quatro indivíduos adultos portadores de deficiência mental. Relações condicionais entre três conjuntos de estímulos semi-representacionais com dois elementos foram ensinadas através dessas tarefas. Inicialmente, dois participantes aprenderam a relação $\mathrm{BA}$, de forma individual e direta, e logo após eles aprenderam a relação CA por observação. Para os demais participantes, CA foi ensinada individual e diretamente, seguida de BA, por observação. Em seguida, testes para verificação da emergência de relações de simetria e transitividade foram aplicados. Um participante mostrou prontamente aprendizagem das relaçôes ensinadas. Os outros três necessitaram de ensino mais extensivo, e dois deles vieram finalmente a aprender as relações ensinadas, através de um procedimento adicional que utilizou instruções específicas. Todos os três participantes que aprenderam as discriminaçôes condicionais de linha de base também demonstraram a emergência das novas relações de discriminaçôes condicionais testadas (BC e CB). Os autores discutiram, dentre outros aspectos, a característica de economia de esforços de ensino do procedimento, uma vez que os participantes aprenderam diretamente a relação entre dois conjuntos de estímulos e, por modelação, aprenderam a relação entre dois outros conjuntos de estímulos, e obtiveram quatro novas relações emergentes. Este foi um aspecto importante dos resultados, pois tratou do fenômeno observado - equivalência de estímulos - obtido através de um procedimento até então original, a aprendizagem observacional. No entanto, pela necessidade de procedimentos adicionais para observação tanto da aprendizagem dos comportamentos de linha de base, quanto para os comportamentos emergentes, os resultados de MacDonald et al. deixaram questóes importantes para serem futuramente investigadas.

Em MacDonald et al. (1986) o procedimento foi aplicado manualmente pelos experimentadores. Uma questão que se coloca com a aplicação de um procedimento complexo não automatizado, é se a interação mantida com o participante se procede sem que o experimentador o induza a responder de maneira pré-determinada. Uma outra questão se coloca sobre a fidedignidade da replicação do procedimento com os diferentes participantes, fundamental para explicar a variabilidade intersujeitos. Com efeito, o procedimento adicional utilizado, que redundou na aprendiza- 
gem de dois dos três participantes, foi insuficientemente descrito para fins de replicação e, portanto, difícil de julgar o quanto dele foi importante na determinação dos resultados. Entretanto, é necessário considerar que para os demais participantes a aprendizagem das relações condicionais por observação somente ocorreu após treino extensivo e procedimentos adicionais, e para um não ocorreu. Estes dados indicam que a aprendizagem observacional de relações condicionais não ocorre tão facilmente para pessoas com necessidades especiais, sendo necessárias mais investigações, sobretudo, para obter maiores esclarecimentos no que se refere à influência de aprendizagem prévia de discriminações condicionais sobre a aprendizagem de relações subseqüentes.

Apesar das questôes metodológicas levantadas acima, o estudo de MacDonald et al. (1986) abriu a possibilidade de investigações sobre aprendizagem observacional de fenômenos não diretamente observados, com metodologia científica consistente, e sugeriu que as pesquisas na área deveriam enfocar a maneira pela qual poderíamos ensinar mais rapidamente indivíduos a aprenderem por observação, e é surpreendente que até o momento ainda não se tenha investido mais nesta área.

$\mathrm{O}$ estudo proposto pretende replicar o procedimento geral de MacDonald et al. (1986) com adultos com desenvolvimento típico, com o objetivo de estender os resultados para outra população e de padronizar o procedimento informatizado de modelação. No presente estudo, portanto, BA foram ensinadas por ação direta de conseqüências diferenciais, e CA por modelação, mantendo-se, assim, o delineamento original de MacDonald et al., e centrando a investigação em outras variáveis como informatização do ensino, o experimentador atuando como demonstrador, uso de estímulos familiares e acréscimo de instruções sobre a observação. Após a fase de ensino, serão testadas as relaçõos emergentes determinantes das propriedades de simetria, transitividade e equivalência. Para finalizar, serão ensinadas as relaçóes DA e EA, por modelação, e testadas as relações emergentes determinantes da expansão da classe de equivalência anteriormente estabelecida.

\section{MÉTODO}

\section{Participantes}

Participaram do estudo seis estudantes universitários dos cursos de Engenharia de Materiais e Biologia da UFSCar. Os participantes eram voluntários, que responderam ao convite verbal feito aos alunos freqüentadores de uma disciplina introdutória de Psicologia para alunos de licenciatura, entretanto, eles não possuíam conhecimento sobre os objetivos do experimento e nem história experimental na área. A participação na pesquisa concedia, ao final do experimento, pontos adicionais na disciplina. Na Tabela 1 são apresentados os participantes por idade e sexo.

\section{Tabela 1}

Descrição dos participantes por idade e sexo.

\begin{tabular}{ccc}
\hline Participante & Idade $^{\mathrm{a}}$ & Sexo \\
\hline \hline P1 & 19 a. $9 \mathrm{~m}$. & $\mathrm{M}$ \\
P2 & 24 a. $4 \mathrm{~m}$. & $\mathrm{M}$ \\
P3 & 21 a. $7 \mathrm{~m}$. & $\mathrm{M}$ \\
P4 & 20 a. $8 \mathrm{~m}$. & $\mathrm{M}$ \\
P5 & 22 a. $10 \mathrm{~m}$. & $\mathrm{F}$ \\
P6 & 25 a. & M \\
\hline
\end{tabular}

${ }^{a}$ Idade no início do experimento. 
Ambiente, Materiais e Estímulos Experimentais As sessões foram conduzidas em uma sala do Laboratório de Aprendizagem Humana, Multimídia Interativa e Ensino Informatizado (LAHMIEI), no Departamento de Psicologia da UFSCar. Cada participante ficou sentado em frente a uma mesa contendo um computador Apple Macintosh Classic, com monitor monocromático de nove polegadas e tela sensível ao toque. O programa computacional para pesquisa Spell (Dube \& McIlvane, 1989) apresentava os estímulos, as conseqüências programadas, registrava as respostas de escolha, e gerava o relatório da sessão em disquete de 1,44 megabytes.

Durante as sessōes estavam presentes o participante $(\mathrm{P})$ e o experimentador (E), no ensino e nos testes individuais; no ensino por modelação, estavam presentes o participante, ao lado deste o demonstrador (D), e o E.

Os estímulos experimentais foram figuras monocromáticas de objetos comuns, selecionadas arbitrariamente, apresentadas da fonte MTSPIX, tamanho 36, e apresentadas na tela do computador em tamanho real de 2,5 $\mathrm{cm} \times 2,5 \mathrm{~cm}$, em média.

$\mathrm{Na}$ Figura 1 apresentam-se os estímulos e um diagrama das relações ensinadas e testadas através da estrutura de treino do estímulo de comparação como nódulo (Green \& Saunders, 1998).

\section{Procedimento Geral}

$\mathrm{O}$ projeto foi inicialmente submetido e aprovado pelo Comitê de Ética de Pesquisas com Seres Humanos da UFSCar.

Escolha de acordo com o modelo. Foram ensinadas relações de discriminação condicional entre cinco conjuntos de estímulos (A, B, $\mathrm{C}, \mathrm{D}$ e E) através de tarefas de escolha de acor-

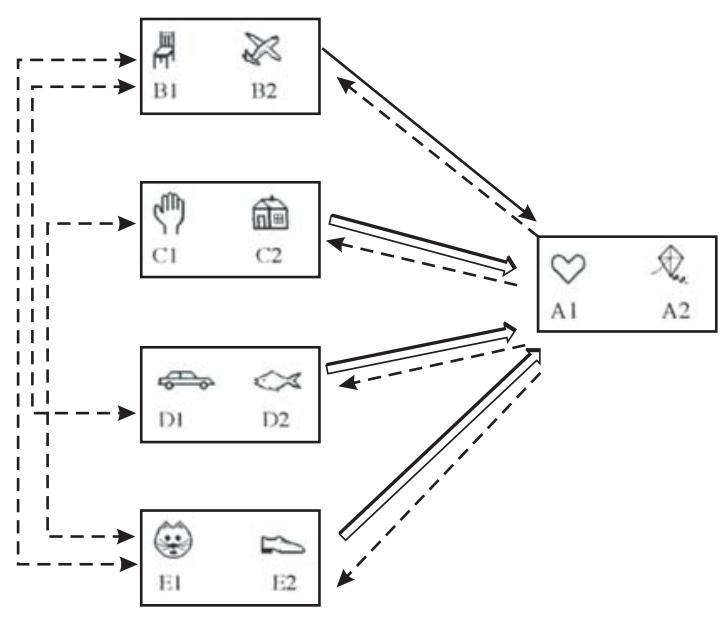

Figura 1. Estímulos e diagrama experimental. Cada retângulo representa um conjunto de estímulos, os quais são designados alfanumericamente com finalidade exclusivamente didática, não sendo visíveis aos participantes. Os conjuntos estão ligados por setas que representam relações condicionais entre os estímulos. As setas apontam do estímulo-modelo para o de comparação. A linha cheia representa o ensino individual; a seta dupla representa o ensino por modelação e os testes individuais das relaçóes de linha de base; e as linhas tracejadas representam os testes das relaçôes

do com o modelo, e com a estrutura de treino do estímulo de comparação como nódulo (CaN) (Green \& Saunders, 1998). Cada conjunto de estímulos era composto por dois membros com denominação alfanumérica (A1A2, B1B2, C1C2, D1D2 e E1E2), para efeitos didáticos (Figura 1).

Cada sessão consistia de número idêntico de tentativas de cada tipo de relação ensinada ou testada, distribuídas de maneira quase randômica. Os estímulos correspondentes à escolha correta não deveriam se localizar na mesma posição por mais de duas tentativas consecutivas, e as mesmas relações não deveriam ser apresentadas por mais de duas tentativas consecutivas. Foram designadas arbitrariamente como corretas as relaçōes entre membros alfanuméricos que possuíam desinência numérica igual, ou seja, o estímulo A1 seria correto na presença do estímulo $\mathrm{B} 1, \mathrm{~A} 2$ na presença de 
B2, e assim por diante, e incorretas quaisquer outras escolhas diferentes dessas. No ensino individual e direto foram apresentadas conseqüências diferenciadas após escolhas corretas e incorretas. As escolhas corretas foram seguidas por um som, emitido pelo computador, e por um efeito, semelhante a um piscar de tela. As escolhas incorretas foram seguidas por uma tela branca, pelo intervalo entre tentativas de $1 \mathrm{~s}$, e por uma nova tentativa. Nas fases de testes as respostas foram seguidas apenas do intervalo entre tentativas. Ao final de cada sessão apresentou-se uma mensagem escrita na tela do computador (p.ex. "Legal”; "Parabéns"; "OK”; e, ao final, "Obrigado pela sua participação").

Ensino por modelação. Após atingir o critério de desempenho nas sessões de ensino individual os participantes eram colocados em dupla com o demonstrador. Durante o ensino por modelação, as sessões continham algumas das relações aprendidas durante o ensino individual intercaladas randomicamente com novas relações que eram realizadas pelo demonstrador. Inicialmente, era dada uma instrução sobre a tarefa (ver detalhes adiante) e, ora o participante, ora o demonstrador, respondiam às suas respectivas tentativas e recebiam consequiências por suas escolhas corretas. As conseqüências eram as mesmas do ensino individual. Mediante critério de desempenho o participante realizava individualmente uma sessão análoga, na ausência do demonstrador. Desta vez, respondia as tentativas já previamente aprendidas e também aquelas que foram observadas durante a realização da tarefa pelo demonstrador, entretanto, não havia conseqüências diferenciadas para suas escolhas, por isso esta sessão foi denominada Testes Individuais. Mediante obtenção de critério de desempenho nas relações observadas e naquelas apren- didas diretamente o participante prosseguia para as demais fases experimentais.

$\mathrm{Na}$ Tabela 2 apresenta-se a síntese das fases experimentais, na seqüência em que foram apresentadas, e as características específicas de cada uma delas, seguida de uma descrição detalhada das fases experimentais.

\section{Fases Experimentais}

O experimento constou de três fases que aplicaram o procedimento de escolha de acordo com o modelo. A primeira ensinou as relações BA, pelo procedimento individual de ensino, e as relações CA, por modelação, seguindo-se dos testes, pelo procedimento individual, para verificação das relações emergentes $\mathrm{BC}$ e $\mathrm{CB}$, de equivalência. A segunda fase constou das relações BA, ensinadas pelo procedimento individual, como linha-de-base (LB), e o ensino das relações DA, por modelação, seguindo-se os testes para verificar a expansão das classes de equivalência, pelo procedimento individual. A terceira fase replicou a fase anterior, com uma nova relação EA, ensinada por modelação, e verificou-se maior expansão das classes de equivalência.

\section{Fase 1}

Ensino individual. Consistiu no ensino direto das relações BA. Foi composto por 24 tentativas de BA (B1A1 e B2A2, em igual número) randomicamente intercaladas. A primeira tentativa iniciou-se com a apresentação do estímulo-modelo (B1) no centro da tela do computador, seguida da instrução "Toque". Após a resposta do participante, dois estímulos de comparação (A1 e A2) foram apresentados na parte inferior da tela, seguidos da seguinte instrução: "Quando aparecer a cadeira (B1) escolha o coração (A1). Quando você acertar o computador irá emitir um som e haverá um contraste figura- 
Tabela 2

Síntese das fases experimentais contendo identificação das relaçôes ensinadas e testadas individualmente (Testes I), das condições de ensino individual (Ensino I) ou por modelação (Ensino M), porcentagem de reforçamento para o participante e critério de desempenho na sessão (porcentagem de escolhas consistentes com a formação de classes).

\begin{tabular}{|c|c|c|c|c|}
\hline Fases & $\begin{array}{c}\text { Relaçōes } \\
\text { (n' }{ }^{\circ} \text { de Tentativas) }\end{array}$ & $\begin{array}{c}\text { Condiçōes } \\
\text { de Ensino ou Teste }\end{array}$ & $\%$ de Reforçamento & Critério de desempenho \\
\hline \multirow{10}{*}{1} & BA (24) & Ensino I & 100 & $\begin{array}{c}90 \% \text { em } 2 \text { sessóes } \\
\text { Consecutivas }\end{array}$ \\
\hline & BA (12) & LB & 100 & $90 \%$ \\
\hline & $\mathrm{CA}(12)$ & Ensino $\mathrm{M}$ & $-\ldots *$ & \\
\hline & BA $1(2)$ & LB & 0 & $90 \%$ \\
\hline & $\mathrm{CA} 12$ & Testes I & 0 & $90 \%$ \\
\hline & $\mathrm{BA}(6)-\mathrm{CA}(6)$ & $\mathrm{LB}$ & 0 & $90 \%$ \\
\hline & $\mathrm{AB}(6)-\mathrm{AC}(6)$ & Simetria & 0 & $90 \%$ \\
\hline & & LB & 0 & $90 \%$ \\
\hline & $\mathrm{BA}(6)-\mathrm{CA}(6)$ & Transitividade & & \\
\hline & $\mathrm{BC}(6)-\mathrm{CB}(6)$ & e Equivalência & 0 & $83,3 \%$ \\
\hline \multirow{10}{*}{2} & $\mathrm{BA}(12)$ & LB & 100 & $90 \%$ \\
\hline & $\mathrm{DA}(12)$ & Ensino $M$ & $\ldots-\ldots *$ & \\
\hline & $\mathrm{BA}(12)$ & LB & 0 & $90 \%$ \\
\hline & DA (12) & Testes I & 0 & $90 \%$ \\
\hline & $\mathrm{BA}(6)-\mathrm{DA}(6)$ & LB & 0 & $90 \%$ \\
\hline & $\mathrm{AD}(12)$ & Simetria & 0 & $90 \%$ \\
\hline & $\mathrm{BA}(16)-\mathrm{CA}(16)-\mathrm{DA}(16)$ & LB & 0 & $90 \%$ \\
\hline & $\mathrm{BA}(8)-\mathrm{CA}(8)-\mathrm{DA}(8)$ & LB & 0 & $90 \%$ \\
\hline & $\mathrm{BD}(6)-\mathrm{DB}(6) \mathrm{CD}$ & Transitividade e & & \\
\hline & (6) - DC (6) & Equivalência & 0 & $83,3 \%$ \\
\hline \multirow{10}{*}{3} & $\mathrm{BA}(12)$ & LB & 100 & $90 \%$ \\
\hline & EA (12) & Ensino $M$ & ....* & \\
\hline & $\mathrm{BA}(12)$ & LB & 0 & $90 \%$ \\
\hline & EA (12) & Testes I & 0 & $90 \%$ \\
\hline & $\mathrm{BA}(6)-\mathrm{EA}(6)$ & LB & 0 & $90 \%$ \\
\hline & $\mathrm{AE}(12)$ & Simetria & 0 & $90 \%$ \\
\hline & $\mathrm{BA}(12)-\mathrm{CA}(12)-\mathrm{DA}(12)-\mathrm{EA}(12)$ & LB & 0 & $90 \%$ \\
\hline & $\mathrm{BA}(6)-\mathrm{CA}(6)-\mathrm{DA}(6)-\mathrm{EA}(6)$ & $\mathrm{LB}$ & 0 & $90 \%$ \\
\hline & $\mathrm{BE}(4)-\mathrm{EB}(4)-\mathrm{CE}(4)$ & Transitividade $\mathrm{e}$ & & \\
\hline & $\mathrm{EC}(4)-\mathrm{DE}(4)-\mathrm{ED}(4)$ & Equivalência & 0 & $83,3 \%$ \\
\hline
\end{tabular}

* $100 \%$ de probabilidade de reforçamento para as escolhas corretas do demonstrador.

fundo na tela”. Após a escolha do estímulo de comparação e apresentação das conseqüências, teve início a segunda tentativa, na qual se apresentou o estímulo-modelo B2. A instrução "Toque" foi apresentada novamente, seguida pela resposta de observação, pela apresentação dos estímulos de comparação e pela instrução "Quando aparecer o aviāo (B2) escolha a pipa (A2). Quando você acertar, o computador irá emitir um som e haverá um contraste figura-fun- do na tela". A partir da terceira tentativa as instruções foram suspensas. A segunda sessão foi conduzida sem instruções sobre a tarefa. $\mathrm{O}$ critério de desempenho foi de $90 \%$ de escolhas consistentes em duas sessões consecutivas.

Ensino por modelação. Consistiu no ensino das relações CA. Foi composto por 12 tentativas das relações CA (C1A1 e C2A2), respondidas pelo demonstrador, randomicamente misturadas a um número igual de tentativas das relaçôes BA, res- 
pondidas pelo participante. A instrução foi a seguinte: "Quando aparecer a cadeira (B1) ou o aviāo (B2), você responde (referindo-se ao participante). Quando aparecer a mão (C1) ou a casa (C2) ele responde" (referindo-se ao demonstrador). O computador irá emitir um som e haverá um contraste figura-fundo na tela quando vocês acertarem. Você (referindo-se ao participante), deve olhar como ele (demonstrador) responde, porque depois você terá que fazer o mesmo sozinho". Todas as escolhas foram seguidas de conseqüências. O critério de desempenho foi de 90\% de escolhas consistentes com formação de classes em uma sessão.

Testes individuais das relações observadas. A sessão consistiu em 24 tentativas contendo 12 tentativas de BA e 12 tentativas de CA, porém com $0 \%$ de reforçamento, sem a demonstração das relações. Antes do início da sessão desta fase, assim como nas demais fases de testes descritas abaixo, a seguinte instrução foi apresentada: " $O$ computador não irá emitir o som e nem o contraste figura-fundo quando você acertar". O critério de desempenho foi de $90 \%$ de acertos, em uma sessão, tanto para as relações BA como para as relações CA. Se o critério de desempenho era atingido, os testes de simetria eram introduzidos; caso contrário, o ensino por modelação era reintroduzido.

Testes de simetria. A sessão de testes de simetria consistiu em 24 tentativas, seis delas para as relações BA e seis para as relações CA, linha de base, randomicamente intercaladas com 12 tentativas de testes ( $\mathrm{AB}$ e $\mathrm{AC})$. $\mathrm{O}$ critério de desempenho foi de $90 \%$ de escolhas consistentes para a linha de base e para cada uma das relações testadas. Após a obtenção do critério de desempenho, os participantes realizaram individualmente os testes de transitividade e de equivalência.
Testes combinados de transitividade e equivalência. Foi composto por 24 tentativas, sendo seis tentativas das relações BA e seis de CA, randomicamente intercaladas com 12 tentativas de testes (BC e CB). O critério de desempenho foi de $90 \%$ de escolhas consistentes para as tentativas de linha de base e $83,3 \%$ para as relações testadas.

\section{Fase 2}

O ensino das relações DA foi análogo ao de CA, com exceção da introdução de testes de retenção das relações de linha de base, descrito abaixo, e do aumento no número de tentativas na sessão de testes de transitividade e equivalência. A composição das sessões, o número de tentativas, e o critério de desempenho nas sessões de ensino por modelação das relações DA, teste individual das relações observadas e testes de simetria foram os mesmos da Fase 1.

Teste individual de retenção das relações de linha de base. Foi apresentado imediatamente antes dos testes de transitividade e equivalência. A sessão foi composta por 48 tentativas, sendo 16 tentativas de cada tipo de relação (BA, CA e DA) intercaladas de maneira quase randômica, com $0 \%$ de reforçamento. O critério de desempenho foi de $90 \%$ de escolhas consistentes para cada um dos tipos das relações (BA, CA e DA). Após a obtenção de critério de desempenho nos testes de retenção o participante realizava testes de transitividade e equivalência. A ausência de critério de desempenho para uma ou várias relações levava o participante a realizar, novamente, o respectivo procedimento de ensino.

Testes de transitividade e equivalência. Foi composto de 48 tentativas, sendo 24 tentativas de linha de base (BA, CA e DA) intercaladas de maneira quase randômica com 24 tentativas de testes (BD, DB, DC, CD), e com 
$0 \%$ de reforçamento. O critério de desempenho foi de $90 \%$ de escolhas consistentes para as tentativas de linha de base e $83,3 \%$ para o total de tentativas de testes.

\section{Fase 3}

O ensino por modelação das relações EA foi análogo ao das relaçôes DA, descrito acima, considerando o número de tentativas, estrutura das sessões, e o critério de desempenho. Os testes das relaçôes observadas, os testes de simetria, os testes de retenção e os testes de transitividade e equivalência seguiram os mesmos padrões da expansão DA.

Teste individual de retenção das relações de linha de base. A sessão foi composta por 48 tentativas, sendo 12 tentativas de cada relação (BA, $\mathrm{CA}, \mathrm{DA}$ e EA) intercaladas de maneira quase randômica, com $0 \%$ de reforçamento. Os encaminhamentos diante da presença ou ausência de retenção seguiram os mesmos padrões descritos na Fase 2.

Testes de transitividade e equivalência. Foi composto de 48 tentativas com 0\% de reforçamento, sendo 24 tentativas de linha de base (BA, CA, DA e EA) randomicamente misturadas a 24 tentativas de testes (BE, EB, CE, EC, DE, ED).

O diagrama contendo as relações ensinadas e testadas nas três fases experimentais encontra-se apresentado na Figura 1.

\section{RESULTADOS}

\section{Fase 1}

Todos os participantes aprenderam as discriminações condicionais BA na primeira sessão, com 100\% de acertos. Uma segunda sessão foi conduzida para atender o critério de desempenho em duas sessões consecutivas (Figura 2). A aprendizagem observacional de CA também ocorreu em uma única sessão de ensino por modelação para cinco participantes, e após duas sessôes para o sexto participante (Figura 2).

Os resultados nos testes de simetria e equivalência encontram-se na Figura 3. Cinco participantes (P1, P3, P4, P5, e P6) apresentaram $100 \%$ de escolhas consistentes nos testes e demonstraram formação de duas classes de estímulos equivalentes, A1B1C1 e A2B2C2 (Figura 3). O desempenho nas tentativas de linha de base desses participantes permaneceu inalterado, em 100\% de acertos para todos os participantes. O resultado discrepante foi de P2 (Figura 3), que mostrou consistência nas relações $\mathrm{BA}$ e $\mathrm{CA}$, de linha de base, e formação da relação simétrica $A B$, mas apresentou $83,3 \%$ de escolhas consistentes nos testes de simetria da relação $\mathrm{AC}$, não tendo, portanto, atingido o critério nesta relação, nem nas relações de equivalência (BC e CB), testadas na sessão seguinte. Após a primeira sessão de testes de equivalência, P2 foi submetido a uma nova sessão de ensino de CA, seguida da sessão de testes das relações CA, em ambas com desempenho dentro do critério. Ao retornar aos testes de simetria, P2 mostrou critério nas relações $\mathrm{AB}$ e AC e, na sessão seguinte, critério nos testes das relações de equivalência.

Fase 2

Todos os participantes aprenderam as novas relações de discriminação condicional DA, ensinadas por modelação, realizando uma sessão de ensino com $100 \%$ de acertos e outra de teste (Figura 4). A linha de base BA manteve-se estável, em 100\% de acertos, para todos os participantes.

Todos os participantes apresentaram $100 \%$ de escolhas consistentes com a formação de simetria (Figuras 5 e 6 ). 


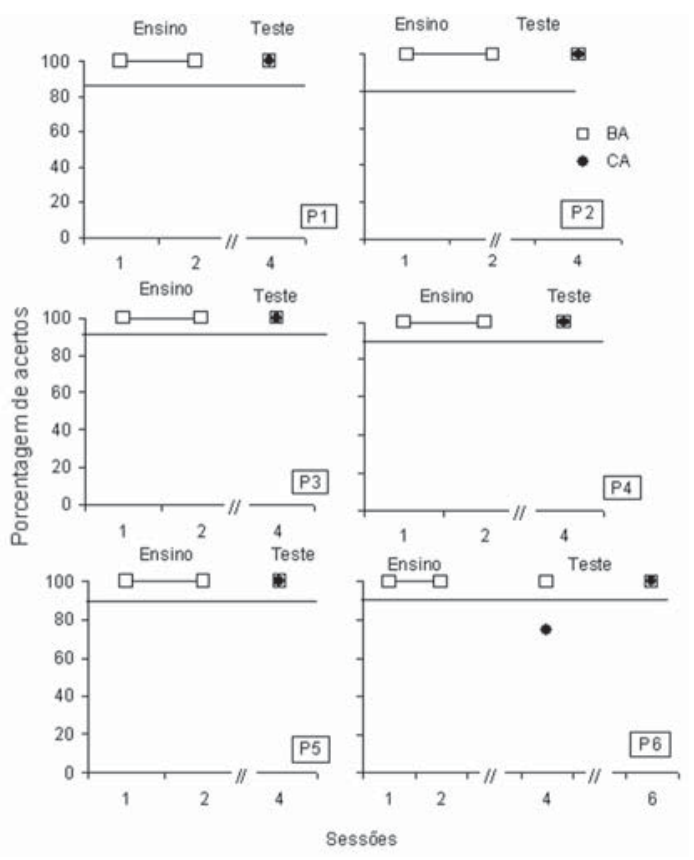

Figura 2. Porcentagem de acertos nas sessões de ensino individual BA e de testes das relações de linha de base. A linha horizontal contínua indica o índice percentual correspondente ao critério de desempenho. As quebras na abscissa, representadas por //, indicam a omissão das sessões de ensino por modelação.

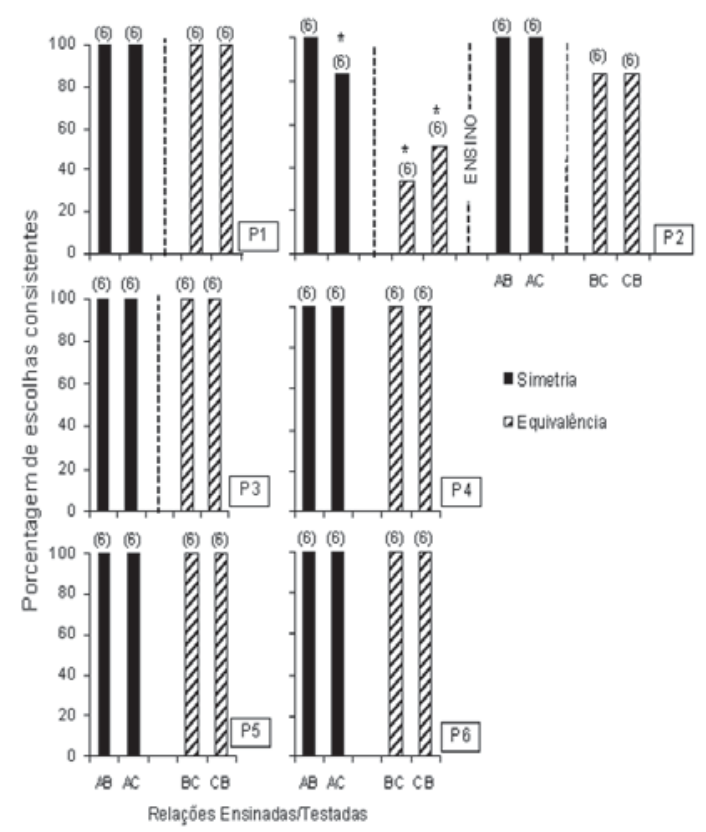

Figura 3. Porcentagem de escolhas consistentes nas tentativas de testes de simetria, transitividade e equivalência para formação de classes para P1, P2, P3, P4, P5 e P6. As linhas tracejadas separam as sessōes de testes. Acima de cada coluna encontrase representado o número total de tentativas, e abaixo, estão indicadas as relações ensinadas/testadas identificadas por conjuntos de duas letras.
No teste de retenção de linha de base, formado pelas relações BA, CA e DA, os P1, P2 e P6 apresentaram desempenho dentro do critério. P3, P4 e P5, no entanto, apresentaram desempenho ligeiramente abaixo do critério nas relaçōes $\mathrm{CA}$. Por essa razão, retornouse ao ensino por modelação das relações CA para esses participantes que, em seguida, receberam uma sessão adicional de testes das relaçōes observadas CA (ver Apêndice A), seguida pelo teste de retenção das relações de linha de base, apresentando 100\% de respostas corretas nessas etapas.

As barras hachuradas das Figuras 5 e 6 apresentam o desempenho dos participantes nas relações de simetria, transitividade e equivalência envolvendo agora os estímulos do conjunto D, e a expansão das classes de equivalência, para A1B1C1D1 e A2B2C2D2. Todos os participantes mantiveram o desempenho na linha de base das relações BA, CA, DA dentro do critério (ver Apêndice B).

Nos testes de transitividade e equivalência todos os participantes, com uma única exceção, apresentaram emergência das relações testadas. P2 não atingiu critério nos testes das relações $\mathrm{BD}$ e $\mathrm{DB}$, mas seu desempenho nos testes DC e CD foi de 100\% de escolhas consistentes com a formação de classes. O desempenho de P2 nas relações BA, CA, DA, de linha de base, permaneceu em $100 \%$ de acertos durante os testes (ver Apêndice B).

\section{Fase 3}

Todos os participantes, com exceção de P2, realizaram a fase de expansão da classe EA e apresentaram desempenho dentro do critério nas relações de linha de base BA e EA do ensino por modelação (Figura 4).

Nos testes de simetria todos os participantes apresentaram critério nas relações de 

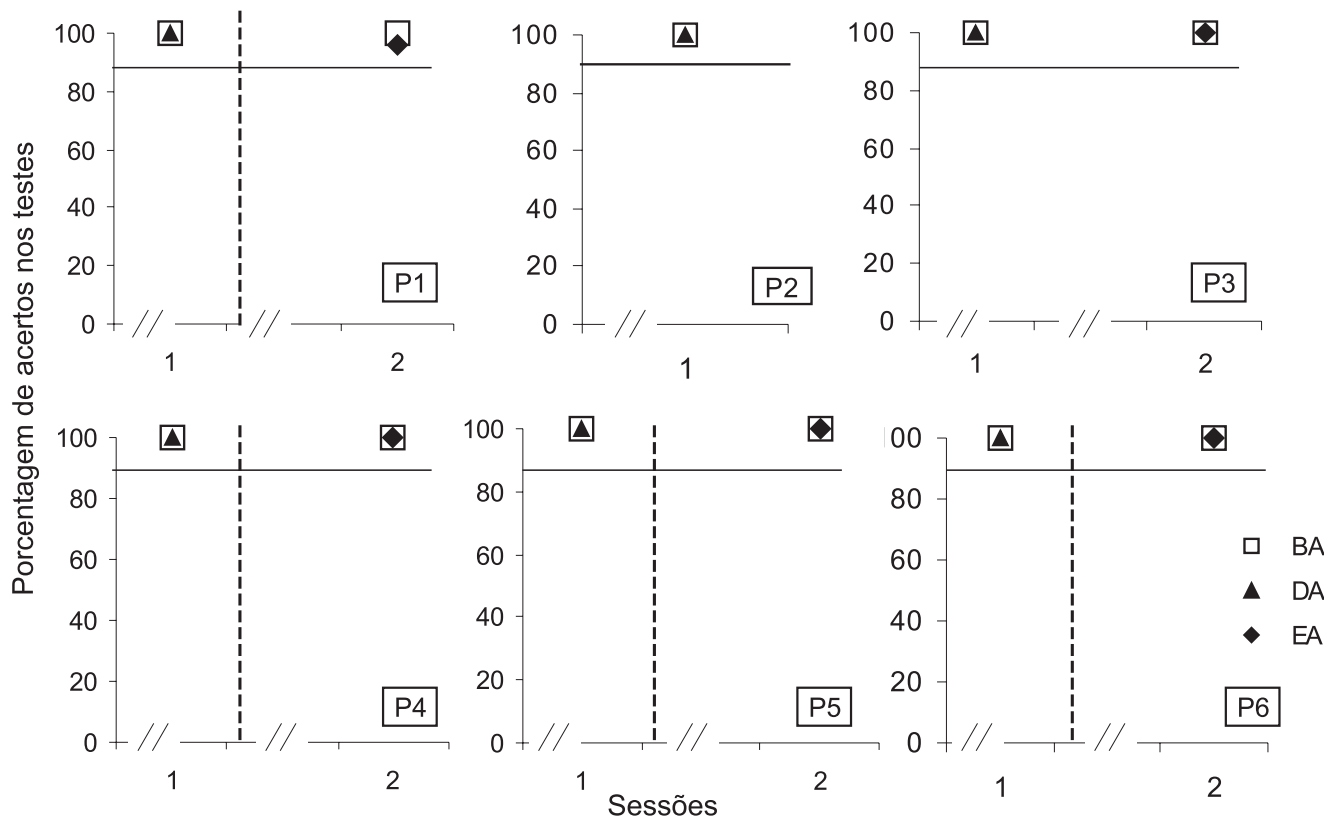

Figura 4. Porcentagem de acertos nas sessōes de testes das relaçōes de linha de base. As linhas tracejadas separam as sessōes das relaçōes DA e EA, respectivamente, Fases 2 e 3 . A linha horizontal contínua indica o índice percentual correspondente ao critério de desempenho. As quebras na abscissa representadas por // indicam a omissão das sessōes de ensino por modelação.

linha de base e nos testes. Nos testes de retenção, todas as relações de linha de base, BA, CA, DA e EA, mantiveram-se consistentes (ver Apêndice C).

Nos testes de transitividade e de equivalência todos os participantes apresentaram desempenhos dentro do critério, indicando a expansão das classes $\mathrm{ABCD}$, para $\mathrm{ABCDE}$, tendo sido os estímulos $\mathrm{C}$, D, e $\mathrm{E}$ incluídos nas classes através de ensino por modelação das relações de discriminação condicional.

\section{DISCUSSÃO}

Adultos com desenvolvimento típico rapidamente apresentaram relações de discriminação condicional também quando essas foram ensinadas através de procedimentos de ensino por modelação. As relaçóes de simetria e equivalência também emergiram prontamente após as relações condicionais iniciais terem sido ensinadas por modelação. Os resultados do presente estudo, portanto, replicaram sistematicamente os de MacDonald et al. (1986) e, adicionalmente, mostraram expansão de classes de equivalência com o procedimento de modelação. O conjunto desses resultados, particularmente os relativos à expansão de classes de equivalência, diferentemente do que haviam considerado Mac Donald et al. (1986), sugere que as variáveis que controlam as características de efeito meramente temporal de aprendizagem observacional podem ser experimentalmente manipuladas e controladas, de maneira teoricamente consistente.

A aprendizagem observacional das discriminações condicionais ocorreu de forma mais rápida do que a relatada por $\mathrm{MacDonald}$ et al. (1986), e esta eficiência pode ter ocorrido devido ao conjunto das diferentes manipulações 


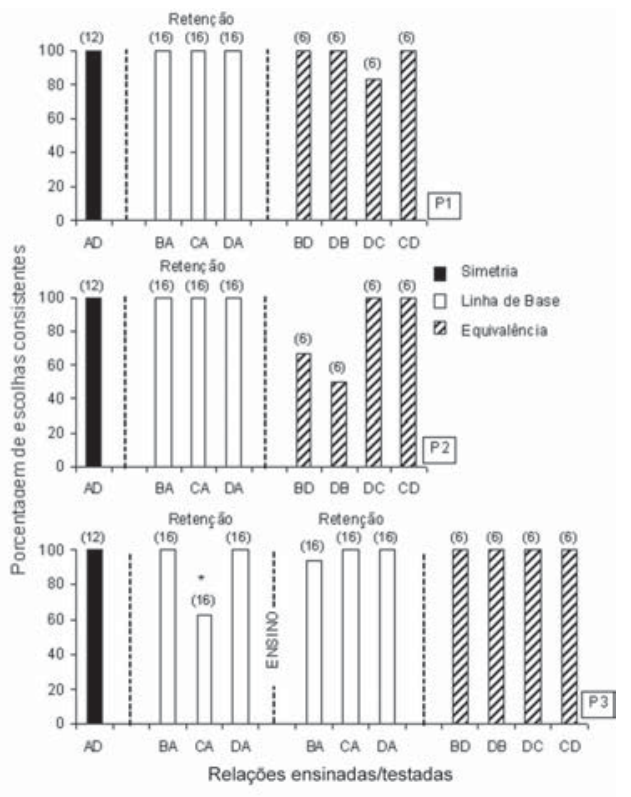

Figura 5. Porcentagem de escolhas consistentes nas tentativas de testes de simetria, de retenção das relaçôes de linha de base, transitividade e equivalência para P3, P4 e P5. As linhas tracejadas separam as sessões de testes. Os asteriscos indicam ausência de critério de desempenho. Os números acima de cada barra indicam o total de tentativas de cada relação e, abaixo, estâo indicadas as relaçốes ensinadas/ testadas identificadas por conjunto de duas letras.

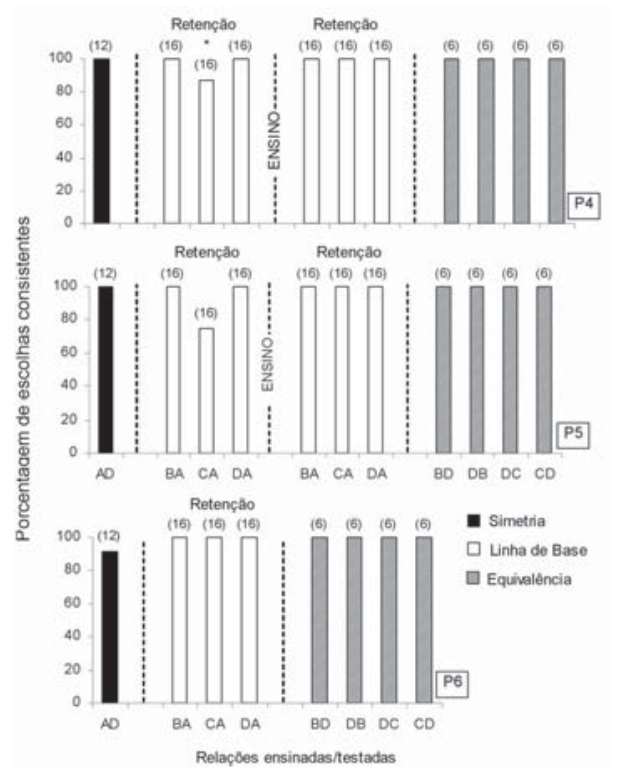

Figura 6. Porcentagem de escolhas consistentes nas tentativas de testes de simetria, de retenção das relaçōes de linha de base, transitividade e equivalência para P1, P2 e P6. As linhas tracejadas separam as sessôes de testes. Os asteriscos indicam ausência de critério de desempenho. Os números acima de cada barra indicam o total de tentativas de cada relação e, abaixo, estão indicadas as relaçôes ensinadas/ testadas identificadas por conjunto de duas letras. experimentais utilizadas, apresentadas em seguida. Pesquisas envolvendo aprendizagem de discriminaçôes condicionais por universitários mostram, de maneira geral, que eles aprendem rapidamente tais relações (ex. Pilgrim \& Galizio, 1995). Estímulos familiares tais como os utilizados no presente estudo, segundo Holth e Arntzen (1998), também facilitam a aprendizagem de discriminaçôes condicionais. A apresentação das tarefas via computador contribuiu para a eficiência do procedimento (Dube $\&$ Mcllvane, 1989) e é fundamental para estudos aplicados. A utilização do experimentador como demonstrador foi uma medida eficiente uma vez que não foi preciso ensinar a relação $\mathrm{CA}$ para um participante exercer tal função, como feito em MacDonald et al. (1986).

Um outro componente importante do procedimento de ensino individual e direto, e potencialmente facilitador da aprendizagem das relaçôes condicionais foi o uso das instruções específicas sobre a tarefa, por exemplo, "quando aparecer a cadeira escolha o coração". Para Sidman (1992, p.21), na instrução, "o estímulo A1 vai com o estímulo B1, e B1 vai com $\mathrm{C} 1$ (...), se o sujeito tem uma história verbal particular, a relação 'vai com' pode já ser uma relação de equivalência”. As teorias de quadros relacionais (Stewart, Barnes-Holmes, Roche, \& Smeets, 2002) e de nomeação (Lowe, Horne, \& Hughes, 2005) também oferecem apoio ao papel facilitador das instruçōes verbais.

A instrução verbal para observação parece ter sido uma variável importante na aprendizagem das discriminaçôes condicionais por observação. Segundo Skinner (1974)

quando mandamos alguém prestar atençāo particular a uma característica do ambiente, nossa ordem é em si mesma um estimulo discriminativo que suplementa o 
estímulo mencionado no controle do comportamento do observador. Oobservadorécondicionado a olhar ou atentar para um estimulo particular quando lhe dizempara 'prestar atençāo' nele, porque nessas condiçōes éreforçado quando faz isso (p.77).

Essas duas últimas características do procedimento muito possivelmente refletem uma limitação de sua aplicabilidade a indivíduos com repertórios verbal e de imitação generalizada bem desenvolvidos. Outras limitações do presente estudo encontram-se apresentadas abaixo.

Ao ensinar discriminações condicionais, MacDonald et al. (1986), assim como o presente estudo, utilizaram conjuntos com dois estímulos cada um, produzindo conseqüentemente uma tarefa de escolha de acordo com o modelo com apenas dois estímulos de comparação. Esta situação pode produzir resultados enviesados, à medida que já existe, inicialmente, $50 \%$ de probabilidade de que o participante emita a escolha correta. Além disso, o participante necessita fazer somente uma escolha baseada na relação entre estímulo condicional e estímulo discriminativo positivo. A outra relação pode ser feita apenas por exclusão, ou escolha do S-, como apontado por Sidman (1987). Embora esta seja uma hipótese a ser considerada, os dados de expansão de classes podem ser um indicativo de que a aprendizagem das relações não ocorreu por exclusão, sobretudo, devido à variabilidade e ao grande número de tentativas de testes conduzidos para verificar a expansão de classes. A aprendizagem de três relações simultâneas e, conseqüentemente, a apresentação de três estímulos de comparação, evita a possibilidade de instalação de alguns desempenhos espúrios.

A correlação observada entre o ensino por observação e a aprendizagem das relações con- dicionais não pode ser tomada como evidência inequívoca de relações funcionais. Ao invés disso, é preciso considerar que, embora a contingência para observação tenha sido um componente importante do ensino, e a aprendizagem possa ter sido facilitada pelo comportamento de observação, os participantes do presente estudo e também os de MacDonald et al. (1986) já haviam aprendido uma relação condicional por ensino individual e direto, anteriormente à aprendizagem de uma discriminação condicional por observação. A aprendizagem destas relações seguintes à primeira, portanto, pode ter ocorrido não somente por modelação, mas em combinação, por ter sido a segunda relação condicional ensinada. Segundo R. Saunders, K. Saunders et al. (1988), a aprendizagem de uma discriminação condicional facilita a aquisição das discriminações seguintes. Para Williams, K. Saunders, R. Saunders, e Spradlin (1995, p.613) "quando apresentamos um problema novo de pareamento arbitrário com duas escolhas, na ausência de conseqüências diferenciais, os sujeitos com experiência prévia em pareamento arbitrário podem mostrar escolhas condicionais consistentes". Além disso, segundo R. Saunders, K. Saunders et al. (1988), R. Saunders, K. Saunders e Spradlin (1990) e também Álvares, Assis, Esteves e Sampaio (2001) e K. Saunders e Spradlin (1990; 1993), as discriminaçôes condicionais estabelecidas quando estão ausentes conseqüências diferenciais podem ter propriedades daquelas estabelecidas quando as conseqüências estão presentes. Dessa for$\mathrm{ma}$, as discriminações condicionais CA que foram aprendidas sem conseqüências diferenciais, através do ensino por modelação, podem ter as mesmas propriedades daquelas ensinadas diretamente, o que justifica a formação e expansão de classes de estímulos obtidas a partir 
de discriminações condicionais aprendidas por observação.

\section{CONSIDERAÇÕES FINAIS}

O presente estudo estendeu os dados de MacDonald et al (1986) e trouxe contribuições acerca da aprendizagem de discriminações condicionais através de um modelo. Em geral, o estudo também ofereceu evidências adicionais que confirmam a robustez do fenômeno de equivalência de estímulos com procedimento de ensino por modelação, que se reafirma como um método para a investigação sistemática do contraste entre ensino individual e ensino por modelação (MacDonald et al.). Desta maneira, como estratégia prática para o ensino em pequenos grupos em ambientes educacionais, a modelação se demonstra uma possibilidade de alternativa promissora para o ensino de tarefas de escolha de acordo com o modelo.

No entanto, pouco o presente estudo avançou no entendimento sobre o papel isolado da aprendizagem por modelação. Investigações futuras terão o propósito de promover a aprendizagem observacional de discriminaçôes condicionais sem história anterior de aprendizagem deste tipo, com procedimento isento de instruções específicas sobre as relações, e apresentação de tarefas com três classes de estímulos. Os novos estudos deverão, ainda, ser conduzidos com crianças em diferentes faixas etárias e com crianças com deficiência mental, para que seja possível identificar os pré-requisitos comportamentais para a aprendizagem observacional de discriminações condicionais e suas reais possibilidades de aplicação prática com essas populações, que muito provavelmente seriam os maiores beneficiários deste tipo de investigação.

\section{REFERÊNCIAS}

Álvarez, S. M. M., Assis, G. J. A., Esteves, I. P., \& Sampaio, M. E. C. (2001). Discriminações condicionais sem conseqüências diferenciais em crianças: efeitos da história de treino precoce de simetria. Estudos de Psicologia, 6 (1), 7-20.

Baer, D. M., \& Sherman, J. A. (1964). Reinforcement control of generalized imitation in young children. American Journal of Mental Deficiency, 22, 498-505.

Browder, D. M, Schoen, S. F., \& Lentz, F. E. (1986). Learning to learn through observation. The Journal of Special Education, 20 (4), 448-461.

de Rose, J. C., de Souza, D. G., \& Hanna, E. (1996). Teaching reading and spelling: exclusion and stimulus equivalence. Journal of Applied Behavior Analysis, 29, 451-469.

de Rose, J. C., de Souza, D. G., Rossito, A. L., \& de Rose, T. M. S. (1989). Aquisição de leitura após história de fracasso escolar: Equivalência de estímulos e generalização. Psicologia: Teoria e Pesquisa, 5, 325-346.

Dube, W. V. \& Mcllvane, W. J. (1989). Adapting a microcomputer for behavioral evaluation of mentally retarded individuals. In J. A. Mulick \& R. F. Antonak (Eds.), Transitions in Mental Retardation. Norwood, NJ: Ablex Publishing Co.

Green, G. \& Saunders, R. R. (1998). Stimulus Equivalence. In: A. Lattal \& M. Perone (Eds.). Handbook of research methods in human operant behavior. (229-261) Nova York: Plenum Press.

Holth, P., \& Arntzen, E. (1998). Stimulus familiarity and the delayed emergence of stimulus equivalence or consistent nonequivalence. The Psychological Record, 48, 81-110.

Lovaas, O., Beberich, B., Perloff, B., \& Schaeffer, B. (1966). Acquisition of imitative speech by schizophrenic children. Science, 151, 705-707.

Lowe, C. F., Horne, P. J., \& Hughes, J. C. (2005). Naming and categorization in young children: III. Vocal tact training and transfer of function. Journal 
of the Experimental Analysis of Behavior, 83, 47-65. MacDonald, R. B. F., Dixon, L. S., \& LeBlanc, J. M. (1986). Stimulus class formation following observational learning. Analysis and Intervention in Developmental Disabilities, 6, 73-87.

Pilgrim, C. \& Galizio, M. (1995). Reversal of baseline relations and stimulus equivalence: I. Adults. Journal of the Experimental Analysis of Behavior, 63, 225238.

Rossit, R., Goyos, C., Araújo, P. M., \& Nascimento, M. H. (2001). Ensino de valores monetários através de escolha de acordo com modelo de componentes e com resposta construída (CRMTS) para adultos portadores de deficiência mental. Resumos de Comunicaçôes Cientificas da Sociedade Brasileira de Psicologia, 53, Rio de Janeiro.

Ruggles, T. R., \& LeBlanc, J. M. (1982). Behavior analysis procedures in classroom teaching. In A. Bellack, M. Hersen, \& A. Kazdin (Eds.). International handbook of behavior modification (959-996). New York: Plenum Press.

Saunders, K. J., \& Spradlin, J. E. (1990). Conditional discrimination in mentally retarded adults: The development of generalized skills. Journal of the Experimental Analysis of Behavior, 54 (3), 239-250.

Saunders, K. J., \& Spradlin, J. E. (1993). Conditional discrimination in mentally retarded subjects: Programming acquisition and learning set. Journal of the Experimental Analysis of Behavior, 60 (3), 571585.

Saunders, R. R., Saunders, K. J., \& Spradlin, J. E. (1990). Long-term stability of equivalence relations in the absence of training or practice. American Journal on Mental Retardation, 95 (3), 297-303.

Saunders, R. R., Saunders, K. J., Kirby, K. C., \& Spradlin, J. E. (1988). The merger and development of equivalence classes by unreinforced conditional selection of comparison stimuli. Journal of the Experimental Analysis of Behavior, 50 (2), 145-162.

Sidman, M. (1971). Reading and auditory-visual equivalence. Journal of Speech and Hearing Research, 14, 5-13.

Sidman, M. (1987). Two choices are not enough. Behavior Analysis, 22, (1), 11-18.

Sidman, M. (1990). Equivalence relations: Where do they come from? In D. E. Blackman \& H. Lejeune (Eds.), Behavior analysis in theory and practice: Contributions and controversies (93-114). Hillsdale, NJ: Erlbaum.

Sidman, M. (1992). Equivalence relations: Some basic considerations. In S. C. Hayes \& L. J. Hayes (Eds.), Understand verbal relations, (15-27). Reno: Context Press.

Sidman, M. (1994). Equivalence relations and behavior: A research story. Boston: Authors Cooperative.

Sidman, M. (2001). Equivalence relations and the reinforcement contingency. Journal of the Experimental Analysis of Behavior, 74 (1), 127-146.

Sidman, M., \& Tailby, W. (1982). Conditional discrimination vs. matching to sample: an expansion of the test paradigm. Journal of the Experimental Analysis of Behavior, 37, (1) 5-22.

Silva, A. M. C. R., Ferreira, P. R., \& Goyos, C. (2001). Escolha de acordo com o modelo com resposta construída (CRMTS): Efeitos na fala de pacientes com troca de fonemas por oposição no traço de sonoridade. Resumos de Comunicaçōes Científicas da Sociedade Brasileira de Psicologia (53), Rio de Janeiro.

Skinner, B. F. (1953/1974). Ciência e Comportamento Humano - Trad. João Cláudio Todorov e Rodolpho Azzi. 2 ed. São Paulo: Livraria Editora Ltda.

Stewart, I., Barnes-Holmes, D., Roche, B., \& Smeets, P. M. (2002). A functional-analytic model of analogy: A relational frame analysis. Journal of the Experimental Analysis of Behavior, 78, 375-396

Striefel, S., \& Wetherby, B. (1973). Instructionfollowing behavior of a retarded child and its controlling stimuli. Journal of Applied Behavior Analysis, 6, 663-670.

Stromer, R., Mackay, H. A. M., Stoddard, L. T. (1992). 
C. GOYOS ET AL.

Classroom applications of stimulus equivalence technology. Journal of Behavioral Education, 3 (2), 225-256.

Williams, D. G., Saunders, K. Saunders, R., \& Spradlin, J. (1995). Unreinforced conditional selection within three-choice conditional discriminations. The Psychological Record, 45, 613-627.
Wright, A. A., Cook, R. G., Rivera, J. J., Sands, S. F., \& Delius, J. D. (1988). Concept learning by pigeons: Matching-to-sample with trial unique video picture stimuli. Animal Learning and Behavior, 16, 436-444.

Encaminhado em 10/1/2005 Aceito em 15/3/2006 


\section{Apêndice A}

Porcentagem de escolhas corretas nas sessões de retorno ao ensino por modelação e testes individuais, realizados após testes de retenção das relaçôes de linha de base.

\begin{tabular}{llccccc}
\hline Relaçóes & P1 & P2 & P3 & P4 & P5 & P6 \\
\hline BA/CA & - & - & 100 & 100 & 95,8 & - \\
BA/CA & - & - & 100 & 100 & 100 & - \\
\hline
\end{tabular}

\section{Apêndice B}

Porcentagem de escolhas corretas nas tentativas de linha de base intercaladas aos testes para as relaçōes emergentes da Fase 2.

\begin{tabular}{cccccccc}
\hline \multirow{2}{*}{ Testes } & $\begin{array}{c}\text { Relaçóes } \\
\text { de LB }\end{array}$ & P1 & P2 & P3 & P4 & P5 & P6 \\
\hline Simetria & BA & 100 & 100 & 100 & 100 & 100 & 100 \\
& DA & 100 & 100 & 100 & 100 & 100 & 100 \\
\hline Transitividade & & & & & & & \\
e equivalência & BA & 100 & 100 & 100 & 100 & 100 & 100 \\
& CA & 100 & 100 & $87,5^{*}$ & 100 & $87,5^{*}$ & 100 \\
& DA & 100 & 100 & 100 & 100 & 100 & 100 \\
\hline
\end{tabular}

* O critério de desempenho na linha de base era calculado pelo total de tentativas de linha de base, independente da relação.

\section{Apêndice C}

Porcentagem de escolhas corretas nas tentativas de linha de base e de testes para as relaçôes emergentes da Fase 3.

\begin{tabular}{ccccccc}
\hline Testes & Relaçóes & P1 & P3 & P4 & P5 & P5 \\
\hline \multirow{5}{*}{ simetria } & BA & 100 & 100 & 100 & 100 & 100 \\
& EA & 100 & 100 & 100 & 100 & 100 \\
& AE & 100 & 100 & 100 & 100 & 100 \\
\hline \multirow{2}{*}{ Retençăo das } & BA & 100 & 100 & 100 & 100 & 100 \\
relaçóes de & CA & 100 & 100 & 100 & 100 & 100 \\
linha de base & DA & 100 & 100 & 100 & 100 & 100 \\
\hline & EA & 100 & 100 & 100 & 100 & 100 \\
& & & & & & \\
& BA & 100 & 100 & 100 & 100 & 100 \\
Transitividade e & CA & 100 & 100 & 100 & $83,3^{*}$ & 100 \\
equivalência & EA & 100 & $83,3^{*}$ & 100 & 100 & 100 \\
& BE & 100 & 100 & 100 & 100 & 100 \\
& EB & 100 & 100 & 100 & 100 & 75 \\
& DE & 100 & 100 & 100 & 100 & 100 \\
& ED & 100 & 100 & 100 & 100 & 100 \\
& CE & 100 & 100 & 100 & 100 & 100 \\
& EC & 100 & 100 & 100 & 100 & 100 \\
\hline
\end{tabular}

* O critério de desempenho na linha de base era calculado pelo total de tentativas de linha de base, independente da relação. 


\section{PROGRAMAS DE PÓS-GRADUAÇÃO STRICTO SENSU EM ANÁLISE DO \\ COMPORTAMENTO NO BRASIL \\ BRAZILIAN GRADUATE PROGRAMS IN BEHAVIOR ANALYSIS}

\section{PROGRAMA DE PÓS-GRADUAÇÃO EM TEORIA E PESQUISA DO COMPORTAMENTO}

\section{DEPARTAMENTO DE PSICOLOGIA EXPERIMENTAL UNIVERSIDADE FEDERAL DO PARÁ}

COORDENADOR: PROF. DR. GRAUBEN ASSIS

Cursos: MESTRADO EDOUTORADO

\section{DOCENTES:}

Carla Cristina Paiva Paracampo

Carlos Barbosa Alves de Souza

Emmanuel Zagury Tourinho

Eleonora Arnaud Pereira Ferreira

Grauben José Alves de Assis

Luis Carlos de Albuquerque

Marcelo Quintino Galvão Baptista

Marcus Bentes Carvalho Neto

Marilice Fernandes Garotti

Olavo de Faria Galvão

Olívia Misae Kato

Romariz da Silva Barros

Informações adicionais: www.ufpa.br/ppgtpc

Contatos: (91) 3201-7662 ou comporta@ufpa.br 